\title{
Eugenia bolampattiana (Myrtaceae), a new species from the Bolampatty Hills of Nilgiri Biosphere Reserve, India
}

\author{
V. Ravichandran, M. Murugesan \& C. Murugan \\ Botanical Survey of India, Southern Regional Centre, TNAU Campus, \\ Lawley Road Post, Coimbatore - 641 003, Tamil Nadu, India \\ taxonmurugesh@gmail.com
}

\begin{abstract}
Eugenia bolampattiana V.Ravich., Murug. \& Murugan (Myrtaceae) is described as a new species from the Bolampatty Hills, Coimbatore District, which is a part of the Nilgiri Biosphere Reserve in the Western Ghats of Tamil Nadu, India. A detailed description, illustration, colour photographs, phenology, and relevant ecological notes are provided, along with a comparison of the morphologically similar species Eugenia mooniana Wight and Eugenia kalamii Shareef et al.
\end{abstract}

Keywords. Critically endangered, endemic, Eugenia sect. Jossinia, Kovai Courtallum

\section{Introduction}

Eugenia L. is the second largest genus in the family Myrtaceae with about 1200 species (Govaerts et al., 2019; Murugesan et al., 2019), which are mainly distributed in South America with fewer species in Africa, Southeast Asia, Malaysia, Madagascar (c. 80 species) and New Caledonia (at least 60 species). The species in Madagascar and New Caledonia comprise c. $12-14 \%$ of the total number of species in the genus (Snow et al., 2015, 2016; Mazine et al., 2016; Giaretta et al., 2018). Molecular phylogenetic studies have indicated that all non-South-American Eugenia are grouped in a single clade currently named Eugenia sect. Jossinia (DC.) Nied. (Mazine et al., 2018). In India, the genus is represented by 26 taxa ( 24 species, one subspecies and one variety), including the recently described Eugenia terpnophylla Thwaites var. keralensis Shareef et al., E. kalamii Shareef et al., E. megamalayana Murugan \& Arum., and E. velliangiriana Murug. et al. All 26 taxa occur in Peninsular India, 20 of which are endemic to the Western Ghats, including three species that are narrowly endemic to the Nilgiri Biosphere Reserve.

\section{Materials and methods}

During a recent botanical exploration in the Bolampatty Hills in the Nilgiri Biosphere Reserve of the Coimbatore District of the Western Ghats, India, the authors came across interesting specimens of a Eugenia species. Critical examination and perusal of the relevant literature (Byng et al., 2015; Duthie, 1879; Murugan, 2002; Gopalan \& 
Srinivasan, 2003; Murugan \& Gopalan, 2005; Gopalan \& Murugan, 2008; Shareef et al., 2011, 2018; Murugan \& Arumugam, 2019; Murugesan et al., 2019), coupled with consultation of type specimens (BM, CAL, E, G, K, L, MH, P), led to the conclusion that the specimens do not match any known species. We therefore propose it here as a new species and provide a photograph and illustration (Fig. 1).

\section{Taxonomic treatment}

Eugenia bolampattiana V.Ravich., Murug. \& Murugan, sp. nov.

The new species is closely allied to Eugenia mooniana Wight, but it differs in the habit being a tree (vs shrub), young shoots fulvous tomentose (vs glabrous), leaf laminas linear lanceolate, base cuneate, apex emarginate (vs ovate, tapering, acuminate), fruits obovoid, c. $1 \mathrm{~cm}$ in diameter, orange (vs globose, c. $1.25 \mathrm{~cm}$ in diameter, pinkish) and number of seeds 1 (vs 1-2). It is also allied to Eugenia kalamii but can be distinguished by being a small tree, 5-12 $\mathrm{m}$ tall (vs undershrub, up to $1 \mathrm{~m}$ tall), young shoots brownish tomentose (vs silvery tomentose), leaf laminas linear lanceolate, base cuneate, apex emarginate (vs ovate-lanceolate, base acute, apex narrowly acuminate), and fruit obovoid to globose, c. $1 \mathrm{~cm}$ in diameter, orange (vs ellipsoid or rarely obovoid, c. 1 $\mathrm{cm}$ in diameter, crimson). (Table 1). - TYPE: India, Tamil Nadu, Coimbatore District, Bolambatty hills, above Kovai Courtallum, 10 $56.216^{\prime} \mathrm{N} 76^{\circ} 41.040^{\prime} \mathrm{E}, 758 \mathrm{~m}, 4$ May 2019, V. Ravichandran \& C. Murugan 145702 (holotype MH; isotypes CAL, MH). (Fig. $1 \& 2$ ).

Medium-sized trees, 5-12 m high; bark smooth, grey; branchlets brown, terete; young stems brownish tomentose. Petiole $0.3-0.5 \mathrm{~cm}$ long, sulcate above, rounded below, sparsely brownish tomentose. Leaf blades $2-5 \times 0.5-1.5 \mathrm{~cm}$, linear-lanceolate or elliptic-lanceolate, membranous, base cuneate, margins entire, apex emarginate, glabrous and glossy above; midrib flat, channelled above, rounded beneath; secondary veins $12-15$ pairs, faint. Flowers usually terminal, rarely axillary, solitary, white (all parts); bracteoles 2, ovate-oblong, $0.8-1 \times 0.8-1.1 \mathrm{~mm}$, densely brown hairy; pedicels $0.3-0.5 \mathrm{~cm}$ long, brownish tomentose, terete; bracts 2 , linear-lanceolate, c. $5 \times 3 \mathrm{~mm}$, densely brownish hairy, glabrous within. Calyx lobes campanulate, lobes $4(2+2)$, unequal, the longer ones c. $1.5 \mathrm{~mm}$, the shorter c. $0.5 \mathrm{~mm}$, densely brownish hairy on lower surface, apex rounded, ciliate. Petals 4, alternate with calyx lobes, ovateelliptic, c. $3 \times 3.5 \mathrm{~mm}$, twice as long as the calyx lobes, often reflexed. Stamens numerous, white; filaments c. $3 \mathrm{~mm}$ long; anthers basifixed, pale white, c. $0.5 \mathrm{~mm}$ long. Hypanthium globose with calyx tube c. $0.5 \mathrm{~mm}$ long; ovary rounded beneath the base of the style and raised above surface of disc, 2-celled, ovules many; style subulate, slender, simple, c. $5 \mathrm{~mm}$ long. Fruits obovoid, rarely globose, c. $2.5 \times 1.5$ $\mathrm{cm}$, calyx lobes persistent but short, orange when ripe. Seeds 1, c. $1 \mathrm{~cm}$, globose to ellipsoid.

Distribution. Known only from the Bolambatty Hills above Kovai Courtallum of the Coimbatore District, Tamil Nadu, India. 

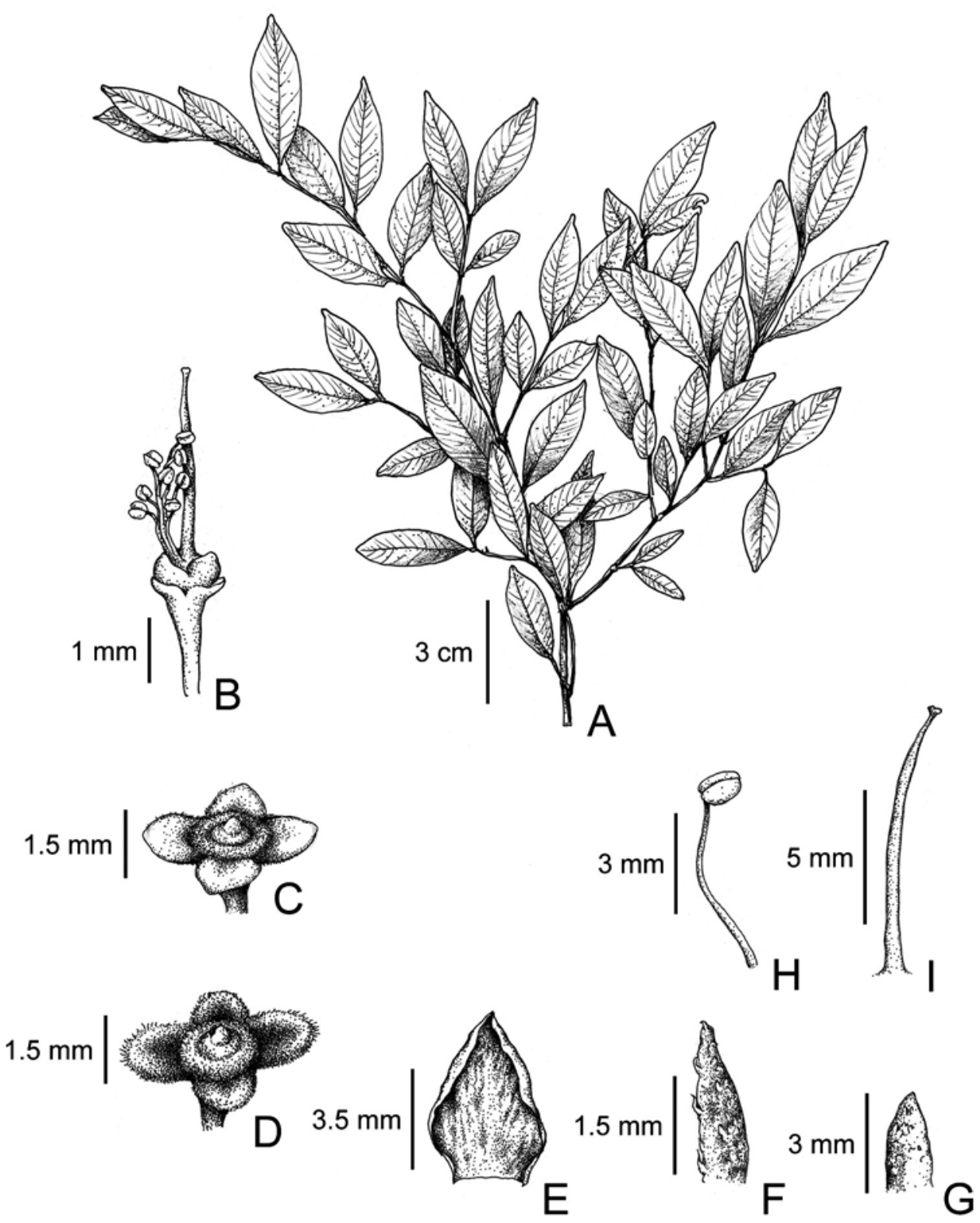

Fig. 1. Eugenia bolampattiana V.Ravich., Murug. \& Murugan. A. Branch. B. Stamens, pistil and disc. C. Calyx and disc (top view). D. Calyx and raised ovary (side view). E. Petal. F. Bract. G. Bracteole. H. Stamen. I. Stigma. Drawn by A.T. Durgadas from V. Ravichandran \& C. Murugan 145702 (Type). 
Table 1. Diagnostic differences between Eugenia bolampattiana and the morphologically similar species E. mooniana and E. kalamii.

\begin{tabular}{|c|c|c|c|}
\hline Character state & E. mooniana & E. kalamii & E. bolampattiana \\
\hline Habit & $\begin{array}{l}\text { Shrubs up to } 4 \mathrm{~m} \\
\text { high }\end{array}$ & Shrubs, up to $1 \mathrm{~m}$ high & $\begin{array}{l}\text { Small trees, 5-12 m } \\
\text { high }\end{array}$ \\
\hline Branchlets & Glabrous & Silvery pubescent & Tawny pubescent \\
\hline Leaves & $\begin{array}{l}4-6 \times 2-3 \mathrm{~cm}, \\
\text { ovate, tapering at } \\
\text { base, apex } \\
\text { acuminate }\end{array}$ & $\begin{array}{l}2.5-9 \times 2.5-3.5 \mathrm{~cm} \text {, } \\
\text { ovate-lanceolate, acute } \\
\text { at base, apex narrowly } \\
\text { acuminate }\end{array}$ & $\begin{array}{l}2-5 \times 0.5-1.5 \mathrm{~cm} \text {, linear } \\
\text { elliptic-lanceolate, } \\
\text { cuneate at base, apex } \\
\text { emarginate }\end{array}$ \\
\hline Lateral veins & 6-10 pairs & 10-14 pairs & $12-15$ pairs \\
\hline Petiole & $\begin{array}{l}6-8 \mathrm{~mm} \text { long, } \\
\text { brown pubescent }\end{array}$ & $\begin{array}{l}1.5-4 \mathrm{~mm} \text { long, silvery } \\
\text { pubescent }\end{array}$ & $\begin{array}{l}3-5 \mathrm{~mm} \text { long, sparsely } \\
\text { brownish pubescent }\end{array}$ \\
\hline Inflorescence & $\begin{array}{l}\text { Solitary, rarely in } \\
\text { terminal clusters or } \\
\text { in short raceme }\end{array}$ & $\begin{array}{l}\text { Terminal, axillary or } \\
\text { rarely lateral }\end{array}$ & $\begin{array}{l}\text { Usually terminal, rarely } \\
\text { solitary in axils }\end{array}$ \\
\hline Pedicel & $\begin{array}{l}0.6-0.9 \mathrm{~cm}, \\
\text { glabrous }\end{array}$ & $\begin{array}{l}0.2-0.6 \mathrm{~cm} \text {, silvery } \\
\text { pubescent }\end{array}$ & $\begin{array}{l}0.3-0.5 \mathrm{~cm}, \\
\text { ferrugineous tomentose }\end{array}$ \\
\hline Fruit & $\begin{array}{l}\text { Globose, diameter c. } \\
1.25 \mathrm{~cm} \text {, pinkish }\end{array}$ & $\begin{array}{l}\text { Ellipsoid or rarely } \\
\text { obovoid; diameter c. } 1 \\
\text { cm, crimson }\end{array}$ & $\begin{array}{l}\text { Obovoid to globose, } \\
\text { diameter c. } 1 \mathrm{~cm} \text {, orange }\end{array}$ \\
\hline Seeds & $1-2$ & 1 & 1 \\
\hline
\end{tabular}

Ecology. It grows in association with Canarium strictum Roxb., Ixora sp., Pavetta tomentosa Roxb. ex Sm., Munronia pinnata (Wall.) W.Theob., Syzygium cumini (L.) Skeels, Litsea glutinosa (Lour.) C.B.Rob., Memecylon umbellatum Burm.f., Litsea stocksii Hook.f., Erycibe paniculata Roxb., Artabotrys zeylanicus Hook.f. \& Thomson, etc. at $758 \mathrm{~m}$ asl.

Phenology. Flowering: April-July. Fruiting: May-July.

Etymology. The new species is named after the type locality, Bolampatty Hills, Tamil Nadu.

Provisional IUCN conservation assessment. The newly described species is currently known only from the type locality, where only 18 individuals occur in a forest area of $10 \mathrm{~km}^{2}$. Its distribution has been evaluated under the Red List Categories and Criteria of the IUCN Standards and Petitions Subcommittee (2017) and is assessed here as 

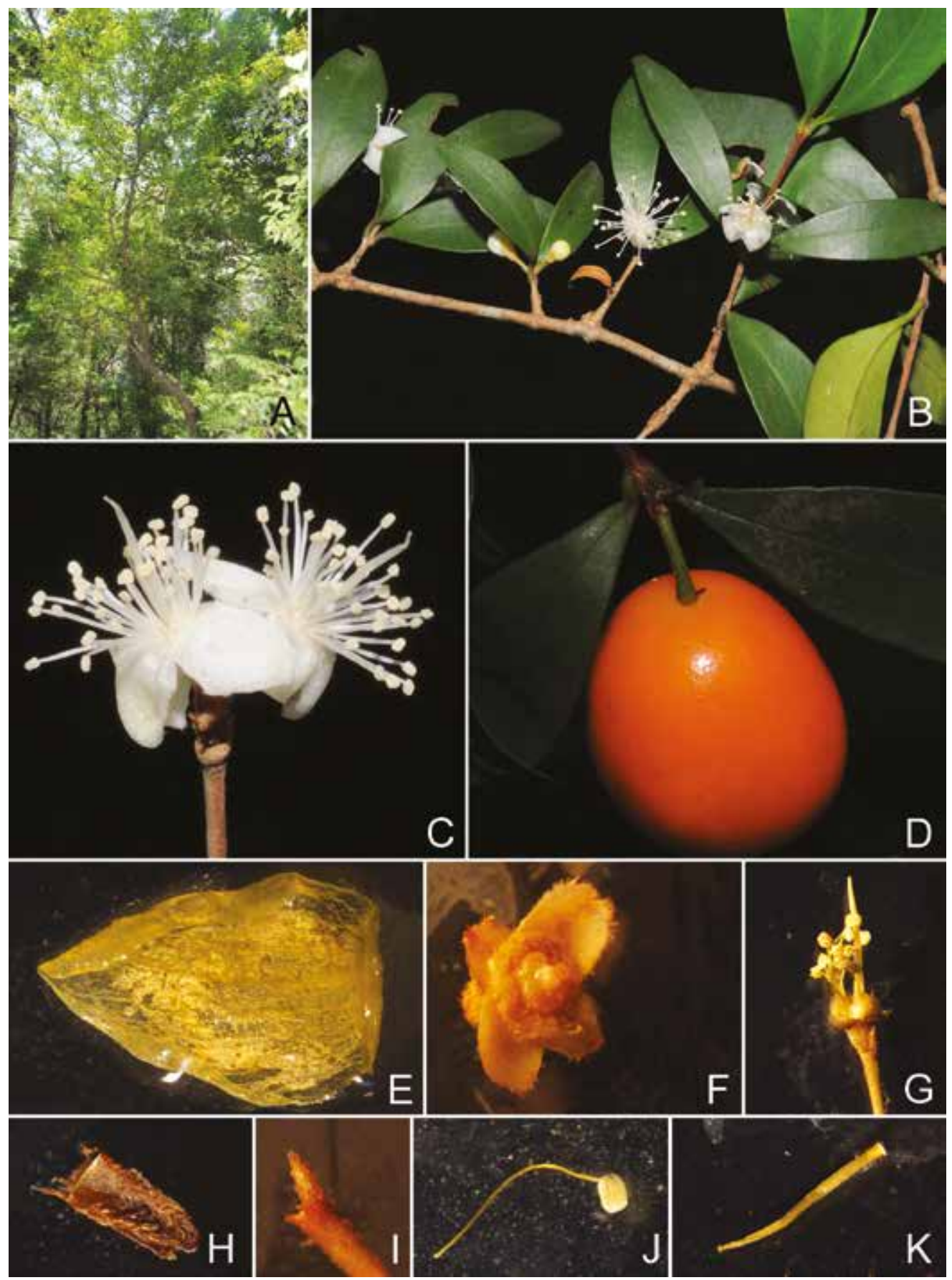

Fig. 2. Eugenia bolampattiana V.Ravich., Murug. \& Murugan. A. Habit. B. Flowering branch. C. Flower, close up view. D. Fruit. E. Petal. F. Calyx lobes with staminal disc. G. Stamens arising from staminal disk and style. H. Bract. I. Bracteole. J. Stamen. K. Stigma. All from V. Ravichandran \& C. Murugan 145702; see Fig. 1 for scale bars. (Photos: V. Ravichandran). 
Critically Endangered (CR B1ab(iii), B2ab(iii)). Habitat destruction caused by tourism, collection of Minor Forest Produces (MFP) by local people, and firewood collection were determined as the major threats during the study period. Extensive exploration of similar habitats and microenvironments in adjacent localities are desirable for determining its greater distribution in other parts of Nilgiri Biosphere Reserve, The Western Ghats.

Additional specimen examined. INDIA: Tamil Nadu: Coimbatore district, Bolambatty hills, above Kovai Courtallum, 1056.216'N 76²41.040'E, 758 m, 4 May 2019, Ravichandran \& Murugan 139167 (MH).

Notes. Eugenia bolampattiana can be distinguished from closely related species using the following key.

\section{Key to Eugenia spp. morphologically similar to Eugenia bolampattiana}

1a. Small to medium sized trees up to $12 \mathrm{~m}$ high E. bolampattiana

1b. Shrubs up to $4 \mathrm{~m}$ high

2a. Branchlets glabrous; leaf veins 6-10 pairs; fruits globose, pinkish ...... E. mooniana

2b. Branchlets silvery pubescent; leaf veins 10-14 pairs; fruits ellipsoid or obovoid, crimson E. kalamii

ACKNOWLEDGEMENTS. The authors express their sincere gratitude to Dr A.A. Mao, Director, Botanical Survey of India, Kolkata for providing facilities and encouragement. The authors are thankful to the Tamil Nadu Forest Department for granting permission to explore the forests. We are thankful to Mr A.T. Durgadas, Artist, BSI, NRC, Dehra Dun and Mr V. Ramesh, Photographer, BSI, SRC, Coimbatore for their excellent contributions to the illustration. The authors are thankful to the reviewers for improving the quality of this manuscript.

\section{References}

Byng, J.W., Wilson, P. \& Snow, N. (2015). Typifications and nomenclatural notes on Indian Myrtaceae. Phytotaxa 217(2): 101-116.

Duthie, J.F. (1879). Myrtaceae. In: Hooker, J.D. (ed.) The Flora of British India, vol. 4, pp. 66-75. London: Reeve \& Co.

Giaretta, A., Souza, M.C., Menezes, L.F.T. \& Peixoto, A.L. (2018). Two new species of Eugenia (Myrtaceae) from the Atlantic forest of Espirito Santo, Brazil. Phytotaxa 336(2): 181-189.

Gopalan, R. \& Murugan, C. (2008). Eugenia agasthiyamalayana (Myrtaceae), a new species from the southern Western Ghats of India. Indian J. Forest. 31: 641-642.

Gopalan, R. \& Srinivasan, S.R. (2003). A new species of Eugenia L. (Myrtaceae) from Seithur Hills, Tamil Nadu, India. J. Bombay Nat. Hist. Soc. 100: 78-80. 
Govaerts, R., Sobral, M., Ashton, P., Barrie, F., Holst, B.K., Landrum, L.L., Matsumoto, K., Mazine, F.F., Nic Lughadha, E. \& Proenca, C. (2019). World checklist of Myrtaceae. Facilitated by the Royal Botanic Gardens, Kew. Published on the Internet. http://wcsp. science.kew.org/. Accessed 28 Jun. 2019.

IUCN Standards and Petitions Subcommittee (2017). Guidelines for Using the IUCN Red List Categories and Criteria. Version 13. Prepared by the Standards and Petitions Subcommittee.

Mazine, F.F., Bünger, M.O., Faria, J.E.Q., Lucas, E. \& Souza, V.C. (2016). Sections in Eugenia (Myrteae, Myrtaceae): nomenclatural notes and a key. Phytotaxa 289(3): 225-236.

Mazine, F.F., Faria, J.E.Q., Giaretta, A., Vasconcelos, T., Forest, F. \& Lucas, E. (2018). Phylogeny and biogeography of the hyper-diverse genus Eugenia (Myrtaceae: Myrteae), with emphasis on E. sect. Umbellatae, the most unmanageable clade. Taxon 67(4): 752769.

Murugan, C. (2002). New species of Xanthophyllum Roxb. (Xanthophyllaceae) and Eugenia L. (Myrtaceae) from Peninsular India. J. Econ. Tax. Bot. 26: 413-418.

Murugan, C. \& Arumugam, S. (2019). Eugenia megamalayana sp. nov. (Myrtaceae)- A new species from the Western Ghats, India. Taiwania 64(1): 4-8.

Murugan, C. \& Gopalan, R. (2005). A new species of Eugenia (Myrtaceae) from the Western Ghats, India. Nordic J. Botany 23: 625-627.

Murugesan, M., Ravichandran, V., Murugan, C.\&Arumugam, S. (2019). Eugenia velliangiriana (Myrtaceae), a new species from the Western Ghats, India. Webbia 73(1): 23-27.

Shareef, S.M., Santhosh Kumar, E.S. \& Roy, P.E. (2011). Eugenia terpnophylla var. keralensis var. nov. (Myrtaceae) from Kerala, India. Nordic J. Botany 29: 455-457.

Shareef, S.M., Santhosh Kumar, E.S., Shaju, T. \& Prakashkumar, R. (2018). Eugenia kalamii (Myrtaceae), a new species from Western Ghats, India. Pl. Sci. Today 5(4): 196-200.

Snow, N., Callmander, M.C. \& Phillipson, P. (2015). Studies of Malagasy Eugenia - IV: Seventeen new endemic species, a new combination, and three lectotypifications; with comments on distribution, ecological and evolutionary patterns. PhytoKeys 49: 59-121.

Snow, N., Dawson, J.W., Callmander, M.W., Gandhi, K. \& Munzinger, J. (2016). New species, new combinations, and lectotypifications in New Caledonian Eugenia L. (Myrtaceae). Candollea 71: 67-81. 
